\title{
REVIEW
}

\section{Keratolytics and Emollients and Their Role in the Therapy of Psoriasis: a Systematic Review}

Arnd Jacobi $\cdot$ Anke Mayer · Matthias Augustin

To view enhanced content go to www.dermtherapy-open.com

Received: August 29, 2014 / Published online: January 21, 2015

(c) The Author(s) 2015. This article is published with open access at Springerlink.com

\section{ABSTRACT}

Introduction: Psoriasis is a common chronic disease with significant impairment in quality of life. As there is no cure, it often requires lifelong disease control to minimize the development of skin lesions and to relieve symptoms. The aim of this publication is to systematically review the role of currently used emollients and keratolytics in the treatment of psoriasis.

Methods: A systematic literature search was conducted in Medline via PubMed regarding reviews, meta-analyses, and trials published from January 1983 to December 2013 dealing with topical administration of emollients and keratolytics in patients with psoriasis. A subsequent search in EMBASE regarding

Electronic supplementary material The online version of this article (doi:10.1007/s13555-015-0068-3) contains supplementary material, which is available to authorized users.

A. Jacobi $(\bowtie) \cdot$ A. Mayer · M. Augustin Institute for Health Services Research in Dermatology and Nursing (IVDP), German Center for Dermatological Research (CeDeF), University Medical Center Hamburg-Eppendorf, Hamburg, Germany

e-mail: a.jacobi@uke.de clinical trials published from 1983 to 2013 was performed to complement the findings.

Results: A total of 60 publications met the inclusion criteria for full-text evaluation. While current reviews, meta-analyses, and guidelines state that adjuvant therapy with emollients and keratolytics should be an obligatory part in the therapy of psoriasis to facilitate descaling and/or penetration enhancement, comprehensive trials on these agents are missing, with the exception of combination products containing salicylic acid and corticosteroids. In the mentioned trials, addition of salicylic acid was beneficial in inducing a more rapid onset of action as well as a reduction of severity parameters and the area affected. However, its use has substantial limitations in young children, in patients with renal/hepatic impairment, with widespread psoriasis, those undergoing phototherapy, or those concomitantly treated with calcipotriene/systemic salicylates.

Conclusion: In view of these shortcomings, there is a need for well-designed studies on suitable keratolytic alternatives to salicylic acid offering an indisputable positive benefit-risk ratio. 
Keywords: Alpha-hydroxy acids; Emollients; Keratolytic agents; Keratolysis; Poly-hydroxy acids; Psoriasis; Salicylic acid; Skin-peeling agents; Skin peeling; Urea

\section{INTRODUCTION}

Psoriasis is a chronic, immune-mediated, inflammatory, systemic disease with predominant skin and joint manifestations. As such, it has significant implications on physical, psychological, and social functioning [1-8]. Psoriasis represents one of the most common chronic inflammatory diseases worldwide, affecting approximately $2-3 \%$ of the population [4, 5, 7, 9-11]. About $80-90 \%$ of patients with psoriasis have the plaque form of the disease $[3,10]$. Although the majority of psoriatic cases are mild to moderate in severity, approximately $20 \%$ of patients suffer from moderate to severe disease as determined by the percentage of body surface area involved or the overall degree of erythema, induration, and desquamation [3]. The impairment in quality of life due to psoriasis has been found to be equivalent to that from major systemic diseases such as arthritis, type 2 diabetes, chronic lung disease, and myocardial infarction [1, 10, 12].

Currently, topical treatments with corticosteroids and vitamin D analogs represent first-line therapies for psoriasis [13]. Topical treatments may also employ calcineurin inhibitors, dithranol, tazarotene, or tar [14]. In patients with more severe or treatment-resistant disease, second- or third-line therapies include phototherapy, systemic agents such as methotrexate, and, more recently, biologicals such as tumor necrosis factor inhibitors. These therapeutic modalities have been proven to be highly effective; however, the potential for long-term toxicity needs to be considered [13].
Phototherapy includes narrow-band ultraviolet (UV) B, broadband UVB, as well as psoralen and UVA photochemotherapy $[15,16]$.

Patient surveys have revealed that, at present, patients do not receive optimal treatments and experience insufficient satisfaction with the efficacy of available topical treatments as reflected by a high rate of non-compliance [17-19]. Since in a cyclical pattern, treatment outcome influences treatment satisfaction, which in turn affects medication adherence; the effectiveness of topical treatments may be improved by increasing patients' adherence [18]. Therefore, and since psoriasis often requires lifelong therapy, safe, convenient, and effective topical regimens with good cosmetic acceptance can be of great benefit in this large patient population [20].

Current guidelines recommend keratolytics such as topical preparations containing urea and salicylic acid as adjuvant therapy for psoriasis and state that these represent an internationally recognized standard in the treatment of all severity states of psoriasis [10]. The claimed benefits of these agents are specified in Table $1[3,4,6,21-23]$.

However, profound and recent evidence on their efficacy from clinical trials is lacking. Therefore, the aim of this publication is to systematically review the role of currently used emollients and keratolytics in the treatment of psoriasis to answer the following questions:

- What do authors of recently published reviews/meta-analyses conclude on the role of emollients and keratolytics in the treatment of psoriasis?

- What is the current evidence from clinical trials on these agents with respect to efficacy and safety that has been published during the last 30 years? 
Table 1 Main objectives of using keratolytics in psoriasis

Main objectives ${ }^{\mathrm{a}}$

- Softening/hydration of the stratum corneum

- Desquamation of hyperkeratotic skin

- Relieve of pruritus/discomfort

- Barrier repair

- Penetration enhancement of topically applied anti-psoriatic drugs/ultraviolet radiation during phototherapy

${ }^{a}$ Cited according to published reviews [3, 4, 6, 21-23]

\section{METHODS}

Systematic literature searches were performed in Medline and EMBASE. Literature searches for relevant articles published within the last 20 years were conducted on December 30, 2013 in Medline via PubMed using the following search term: “(1993/01/01:2013/11/ 30[dp] OR 1993/01/01:2013/11/30[edat]) AND psoriasis AND (keratolysis OR keratolytic OR salicylic acid OR urea)". The results were further confined using specific qualifier words regarding efficacy, safety, or type of publication, for example, "therapeutic use", "efficacy", "effectiveness", "safety", "tolerability", "toxicity", "review", "metaanalysis", "practice guideline", "clinical trial", or "randomized controlled trial". The search yielded a total of 798 articles for consideration. All hits were reviewed based on abstracts and/or Medical Subject Headings (MeSH) terms. Articles that did not specifically deal with the safety and efficacy of emollients or keratolytics in the treatment of psoriasis but dealt with other psoriasis treatments such as hydroxyurea or topical retinoids, with other indications or other topics or subjects (e.g., methods papers, non-clinical, mechanistic studies) were excluded. This applied to the majority of retrieved publications (approximately 90\%). Because salicylic acid is frequently added to dithranol for the prevention of dithranol oxidation, all publications on the combination of salicylic acid and dithranol were excluded [24]. The same applies to efficacy trials conducted in healthy volunteers or in patients with disorders other than psoriasis. Whereas clinical studies investigating keratolytics vs. baseline, placebo, no treatment, or other keratolytics and combination therapy vs. the respective monotherapy without penetration enhancement were considered eligible for inclusion, articles that dealt with the safety or efficacy of conventional keratolytics in combination therapies for psoriasis but did not investigate the monosubstances and did thus not allow drawing conclusions about their specific effect were not considered (approximately $2 \%$ of hits). Articles that were not written in English or German were excluded (approximately $2 \%$ of hits). Guidelines or reviews which had already been revised were not considered. Since the number of articles providing evidence on the role of conventional keratolytics in the therapy of psoriasis published in the last 20 years was limited, the search period was extended to the last 30 years.

The searches for relevant literature published between 1983 and 1993 were conducted in Medline via PubMed using the following search terms: "psoriasis AND (keratolytic[Majr] OR salicylic acid[Majr] OR urea[Majr])", "psoriasis AND (keratolysis OR keratolytic) AND (rct OR randomized controlled trial)" and "psoriasis AND (salicylates[Majr])". Using the above-mentioned selection criteria, a total of 53 guidelines, metaanalyses, reviews, and clinical trials published in German or English language from January 1983 to December 2013 and dealing with topical administration of keratolytics in patients with psoriasis were regarded as potentially relevant 
and selected for full-text assessment. After fulltext review, seven articles were excluded as they did not refer to emollients or keratolytics $(n=2)$ or did not compare combination therapy vs. the respective monotherapy without penetration enhancement $(n=5)$.

Since Medline may not have revealed all relevant publications (i.e., clinical trials), additional searches for original literature on psoriasis published in English or German language between 1983 and 2013 were performed in EMBASE using the qualifier "clinical trial" and the controlled terms "keratolytic agent", "salicylic acid", and "urea". These searches yielded a total of 41 articles for consideration, of which 3 articles had not previously been identified in Medline. The selection was further complemented by publications that were cited in the articles identified by the systematic literature searches and by including relevant current guidelines on psoriasis treatment as well as references therein $(n=11)$.

The results section of this paper consists of four subsections referring to the group of emollients and major keratolytic agents, each starting with a paragraph that contains information on the mechanism of action and evidence of the efficacy and safety as extracted from reviews, meta-analyses, and/or guidelines. This information is followed by the data on efficacy and safety derived from published clinical trials. This review is based on previously conducted studies and does not involve any new studies of human or animal subjects performed by any of the authors.

\section{RESULTS}

From systematic literature searches conducted in Medline via PubMed and EMBASE, a total of
49 publications met the inclusion criteria for this review. Furthermore, the reference lists of included publications were scrutinized for additional studies published in January 1983 or later, adding 11 publications to the selection. Thus, a total of 60 publications were reviewed.

\section{Emollients}

Emollients induce an occlusive film that limits evaporation of water from deeper parts of the skin and allows the stratum corneum to rehydrate itself. The application of natural moisturizing factors induces an increase in the water-binding capacity of the stratum corneum [21]. Regular application of emollients improves comfort and reduces scaling, fissuring [1], and itching in patients with plaque or scalp psoriasis $[25,26]$. Additionally, emollients may increase the efficacy of topical corticosteroids by improving the penetration through the skin layers $[21,27,28]$. Guidelines of care for the management of psoriasis and psoriatic arthritis state that when used as a control in topical steroid trials, non-medicated topical moisturizers demonstrated a response rate ranging from 15 to $47 \%[21,27]$. This broad range may reflect the great variability of their composition; however, in the corresponding guideline dealing with topical therapies, the individual composition of the moisturizers has not been specified [7]. Emollients are usually well tolerated [23]. However, they can cause side effects such as irritant dermatitis, allergic contact dermatitis, fragrance allergy or allergy to other constituents, stinging, cosmetic acne, and pigmentary disorders [21]. In addition, some reports indicate a potential penetrationenhancing effect of the stratum corneum to irritants after long-term use [21]. In clinical and experimental settings it has been shown that 
some emollients, e.g., oil-in-water emollient enhance the penetration of UVA or UVB when used before irradiation, thus increasing the efficacy of phototherapy [29, 30]. However, in vitro studies and studies in healthy volunteers also demonstrated a blocking effect of other emollients, such as white petrolatum [31] and the emollient cream Eucerin (Beiersdorf Inc., Wilton, CT, USA), consistent with their thickness [32].

While 3 small clinical trials including a total of 111 patients provide limited evidence that, compared to baseline, emollients used as a monotherapy may improve skin hydration, barrier function, as well as proliferation and differentiation markers in patients with psoriasis [11, 33, 34], the clinical response showed only a slight symptomatic improvement of psoriasis [33]. Due to inconsistent results from randomized controlled trials, there is limited evidence of a steroid-sparing effect of emollients when used in combination with corticosteroids. In the evaluated studies, only two adverse events of pruritus and folliculitis (each $n=1$ ) were reported (see Table 2) [11, 33-37].

\section{Urea}

Urea is known to exert proteolytic, keratolytic, hydrating, hygroscopic, penetrationenhancing, epidermis-thinning, and antipruritic effects. The moisturizing action of urea in dry and scaly skin conditions has been widely studied and is well accepted [21, 38]. Fluhr et al. [21] suggested that lipid biosynthesis may be increased by topical application of highly concentrated urea. In vitro and in vivo data showed a reduction of DNA synthesis in the cells of the basal layers (by approximately $45 \%$ ), a thinning of the epidermis (by approximately 20\%), a reduction of the epidermal cells, and a prolongation of the generation time of postmitotic epidermal cells $[21,39]$. Therefore, urea has been shown to reduce epidermal hyperproliferation and to induce cell differentiation [27]. As a mechanism of action it has been hypothesized that urea may break hydrogen bonds and interfere with the quaternary structure of keratin thus dispersing and denaturizing keratin without disrupting the epidermal water barrier. Pre-treatment or concomitant treatment with urea may also enhance the efficacy of other topical therapies [39]. Due to its safety, urea-containing preparations represent the standard in the adjuvant therapy of juvenile psoriasis [5]. Only non-systemic side effects have been reported, with mild irritation being the most common, making urea a safe and well-tolerated topical drug [39].

While a small, older comparative study found no statistical differences on severity parameters such as scaling, erythema, and infiltration compared to the vehicle [40], in another trial using the same preparation, treatment for 1 week led to a statistically significant improvement of scaling compared to the vehicle (see Table 3) [38, 40-46]. This is in line with two further studies in which severity parameters of psoriasis such as scaling and/or induration were reduced with urea in monotherapy [39]. Of these, in a small randomized, double-blind study, addition of $10 \%$ urea to the vehicle was significantly more effective regarding epidermal proliferation, stratum corneum hydration, and epidermal thickness with a $60 \%$ reduction of the clinical psoriasis severity score of scaling and a $32 \%$ reduction of induration (see Table 3) compared to the vehicle alone [38]. The ointment base also improved psoriasis, but urea was significantly more effective showing a 40\% reduction in epidermal proliferation compared 


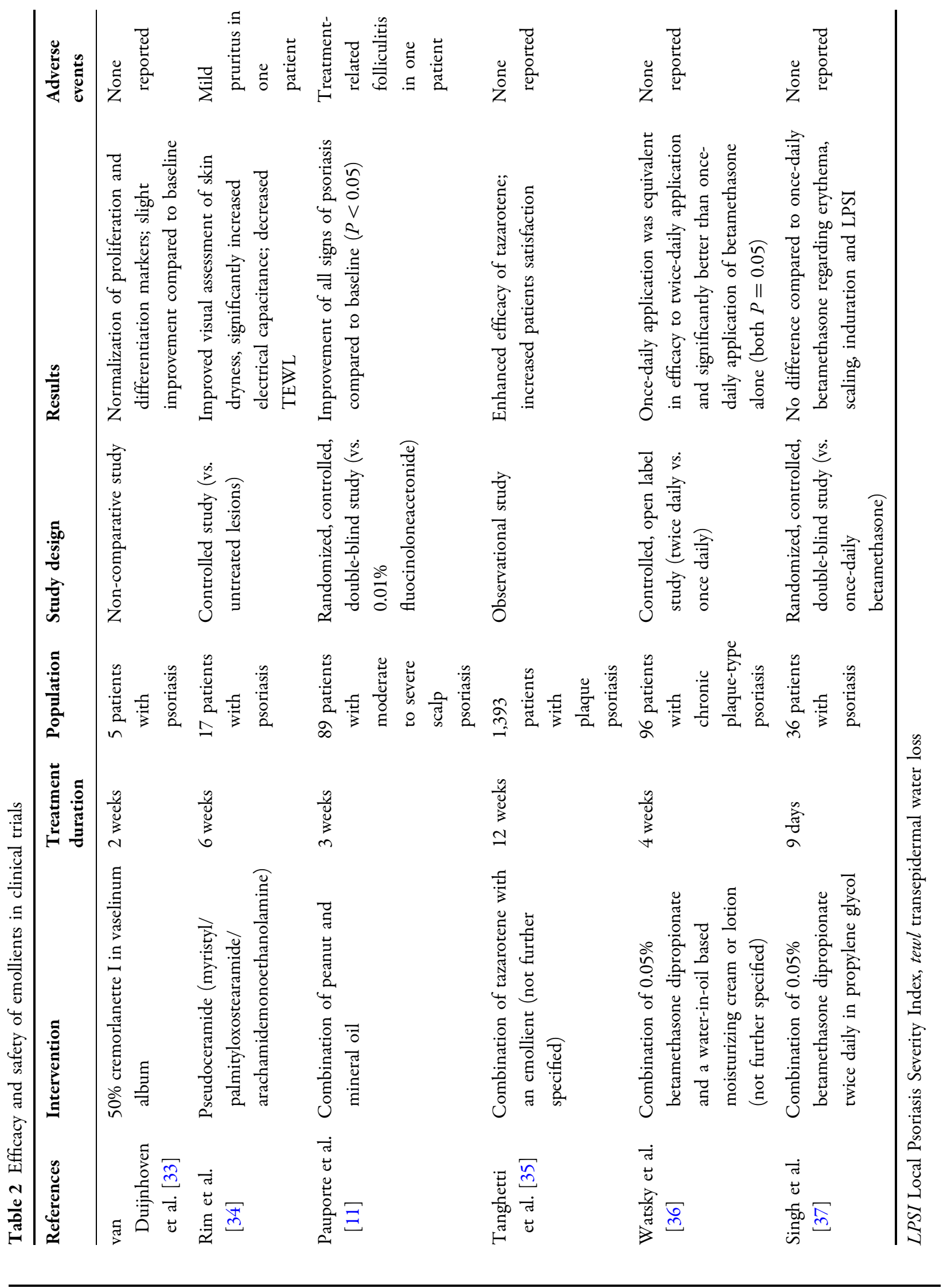




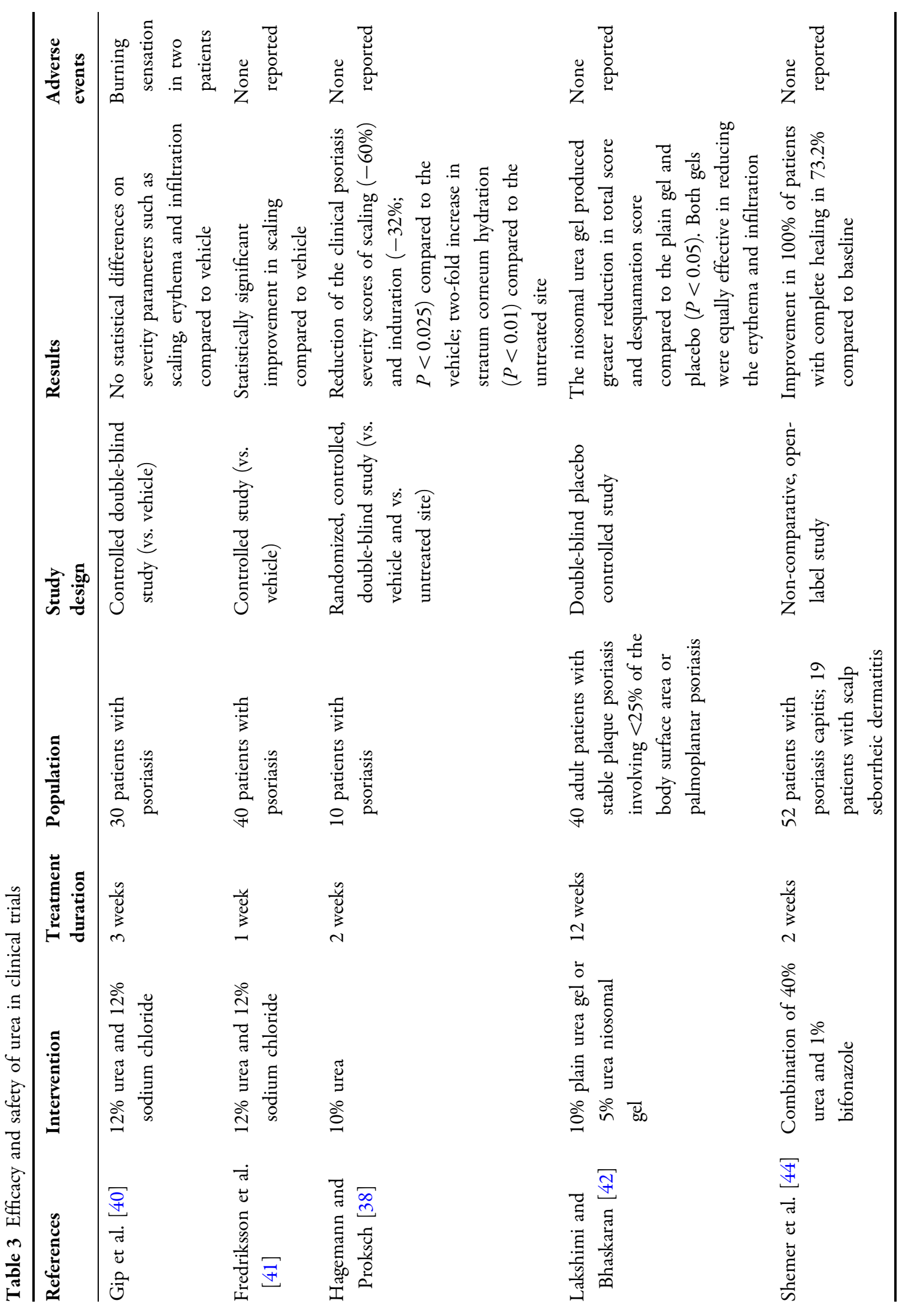




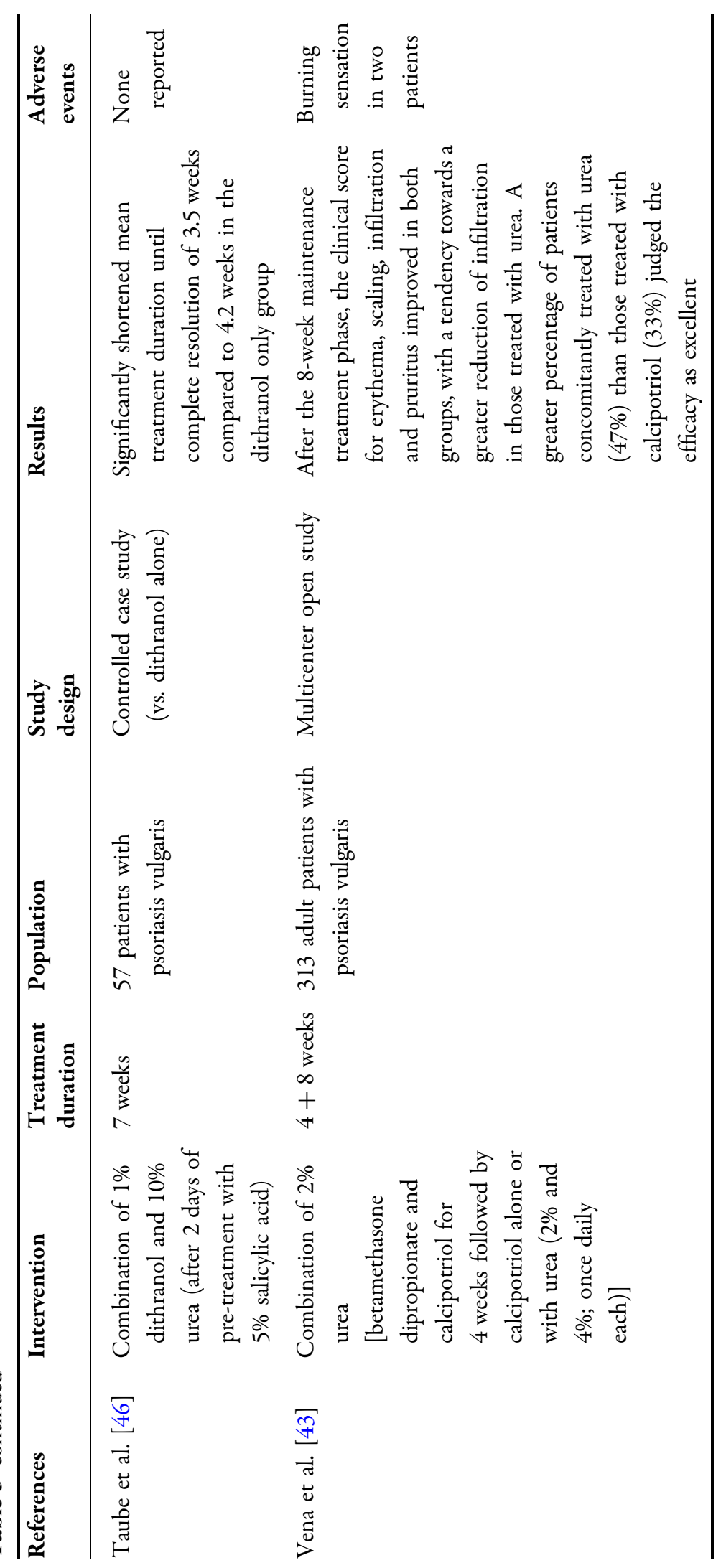


to the vehicle [38]. Similar results were demonstrated in a double-blind, placebocontrolled study, in which two urea gel formulations (10\% plain urea gel or $5 \%$ urea niosomal gel) produced a reduction in erythema, infiltration, and desquamation, as well as in the total psoriasis area and severity index (PASI) score, with the 5\% urea niosomal gel being more effective regarding desquamation compared to the plain gel and placebo. However, the percentage of affected area was not influenced [42]. From three small studies, limited evidence is known for an enhanced efficacy of other topical agents such as bifonazole, dithranol or betamethasone dipropionate and calcipotriol leading to a shortened treatment duration (see Table 3) [43-45]. In the evaluated clinical studies, a burning sensation was observed in three patients (see Table 3) [39, 42].

\section{Alpha-Hydroxy Acids and Poly-Hydroxy Acids}

Alpha-hydroxy acids (AHAs), most notably glycolic acid and lactic acid, are used in chemical-peel solutions to exfoliate thickened skin in hyperkeratotic conditions. However, their use in the treatment of psoriasis has only recently been advocated and, thus, they are not mentioned as keratolytic agents in relevant psoriasis guidelines $[7,17]$. AHAs penetrate the epidermis, inducing an increase in stratum corneum turnover and cause desquamation of the outermost layer without impairing barrier function [21]. They reduce intercorneocyte bonds by increasing the distance between corneocytes due to increased stratum corneum water content, by reducing the charges on the surface of cells, by inhibiting enzymes involved in the cohesion between corneocytes, and by breaking desmosomes as they diminish the $\mathrm{pH}$ of the medium. This reduction can also degrade keratinocytes directly, thus promoting cell proliferation. In the dermis, AHAs can stimulate the biosynthesis of glycosaminoglycans, collagen, and other substances, which cause thickening of the skin [47]. Poly-hydroxy acids (PHAs) also aid in dermal expansion and can provide the components for glycosaminoglycans. PHAs have anti-oxidant and moisturizing properties and may provide protection of psoriatic skin with a disrupted skin barrier. When used in combination with topical corticosteroids, AHAs or PHAs exert a synergistic effect and reduce steroid-induced skin atrophy [3].

For AHAs and PHAs, results from 3 small clinical studies including 57 patients in total are available. One of these trials, a small, controlled study, demonstrated that glycolic acid in monotherapy may lead to significant reduction in hyperkeratosis and erythema as compared to baseline, which was comparable to that achieved with $0.05 \%$ betamethasone valerate (see Table 4) [3, 47, 48]. In a small, short-term study, AHA/PHA and salicylic acid exhibited similar efficacy with regard to the reduction of scaling when compared to baseline (see Table 4) [3]. The third small study found a synergistic effect of $10 \%$ glycolic acid in combination with topical corticosteroids leading to reduced treatment duration and an increased healing rate (see Table 4) [48]. In the evaluated studies, no treatment-related adverse events were reported (see Table 4).

\section{Salicylic Acid}

Salicylic acid is the most commonly used and most thoroughly studied of the currently known keratolytic compounds [21]. In concentrations of 5\% and above, it exerts an increasingly potent, rapid, and deep keratolytic 


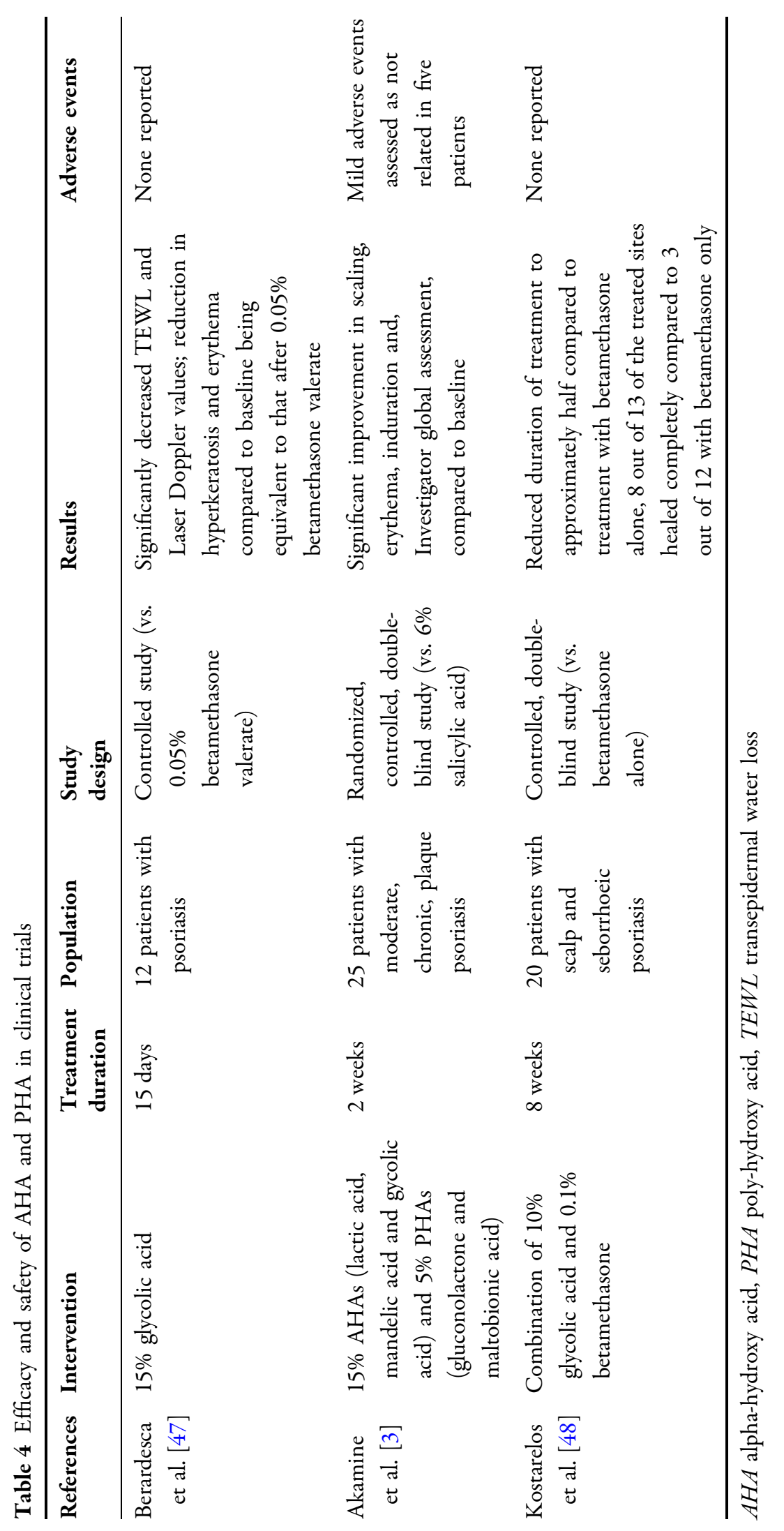


effect on the stratum corneum which leads to descaling [31]. As a mechanism of action, it is suggested that salicylic acid reduces intercellular cohesion between corneocytes by dissolving the intercellular cement material and reducing the $\mathrm{pH}$ of the stratum corneum, thereby increasing hydration and softening [3, $7,31]$. Topical salicylates also reduce pruritus [26] and, at concentrations of $\geq 0.3 \%$, they possess bacteriostatic and bactericidal activity against yeast and Gram-negative and Grampositive bacteria [22]. In their clinical guide, Naldi and Rzany [9] state that there is consensus that salicylic acid is an effective initial and adjunctive treatment in chronic plaque psoriasis. It is most beneficial in extremely thick or scaly psoriatic plaques [22]. According to current guidelines, salicylic acid promotes skin availability of other topical therapies including topical corticosteroids due to its keratolytic and penetration-enhancing effect $[1,7,14,20,49]$. This leads to improved efficacy of other topical treatments. While salicylic acid is commonly applied and effective when used as a pre-treatment before phototherapy $[15,16]$, topically administered salicylic acid, in concentrations $\geq 0.1 \%$, is photoprotective [31]. Thus, application of salicylic acid before UVB phototherapy is not recommended $[15,16,22,32,50,51]$. According to the evaluated reviews, topical use of salicylic acid is limited by the risk of chronic or acute systemic intoxication [2, 7] with symptoms such as oral pain, headache, central nervous system symptoms, dizziness, metabolic acidosis, tinnitus, nausea, vomiting, and gastric symptoms, as well as hyperventilation $[2,3,52]$. These symptoms may occur after prolonged topical treatment of large body surfaces (i.e., $>20 \%)[6,7,25]$, especially in children under 12 years of age and in patients with significant renal or hepatic impairment $[2,3,7,22,25]$.
Local irritation such as stinging, burning, dry skin, peeling, scaling, or contact dermatitis was also reported $[1,9,53]$. Temporary shedding of telogen hair has been observed when used in the treatment of scalp psoriasis [26]. Concomitant use of other drugs, which may contribute to elevated serum salicylate levels, should be avoided [7]. There is an increased risk for developing toxicity to other topically applied agents used concurrently, as salicylic acid may increase their skin penetration [3]. In addition, topical use of salicylic acid can reduce the efficacy of calcipotriol [2].

\section{Monotherapy}

Regarding monotherapy, three studies on salicylic acid including 65 patients were evaluated [54-56]. Of these, an open-label, pilot study demonstrated that $6 \%$ salicylic acid used as a monotherapy was highly effective, well tolerated, and acceptable in 10 patients with scalp psoriasis [54]. All psoriasis severity parameters as well as the percentage of affected area were reduced with a significant decrease in Psoriasis Scalp Severity Index score from 15.3 to 3.0 after 4 weeks of monotherapy when compared to baseline $(P<0.001)$. A total of $60 \%$ of patients were either "completely cleared" or "almost cleared" (see Table 5) [54]. In a randomized, double-blind study in 25 patients investigating $6 \%$ salicylic acid vs. $20 \%$ AHA/PHA cream (see also Table 4), both topical agents resulted in significant improvement of scaling, erythema, and induration, as well as investigator global assessment, at the end of week 1 and 2 and exhibited similar efficacy in reducing scaling. However, the extent of affected area was not investigated in this study (see Table 5) [3]. Similar results were obtained in an earlier, randomized controlled trial reporting successful treatment of moderate or severe scalp psoriasis with $6 \%$ salicylic acid in 30 patients, including 


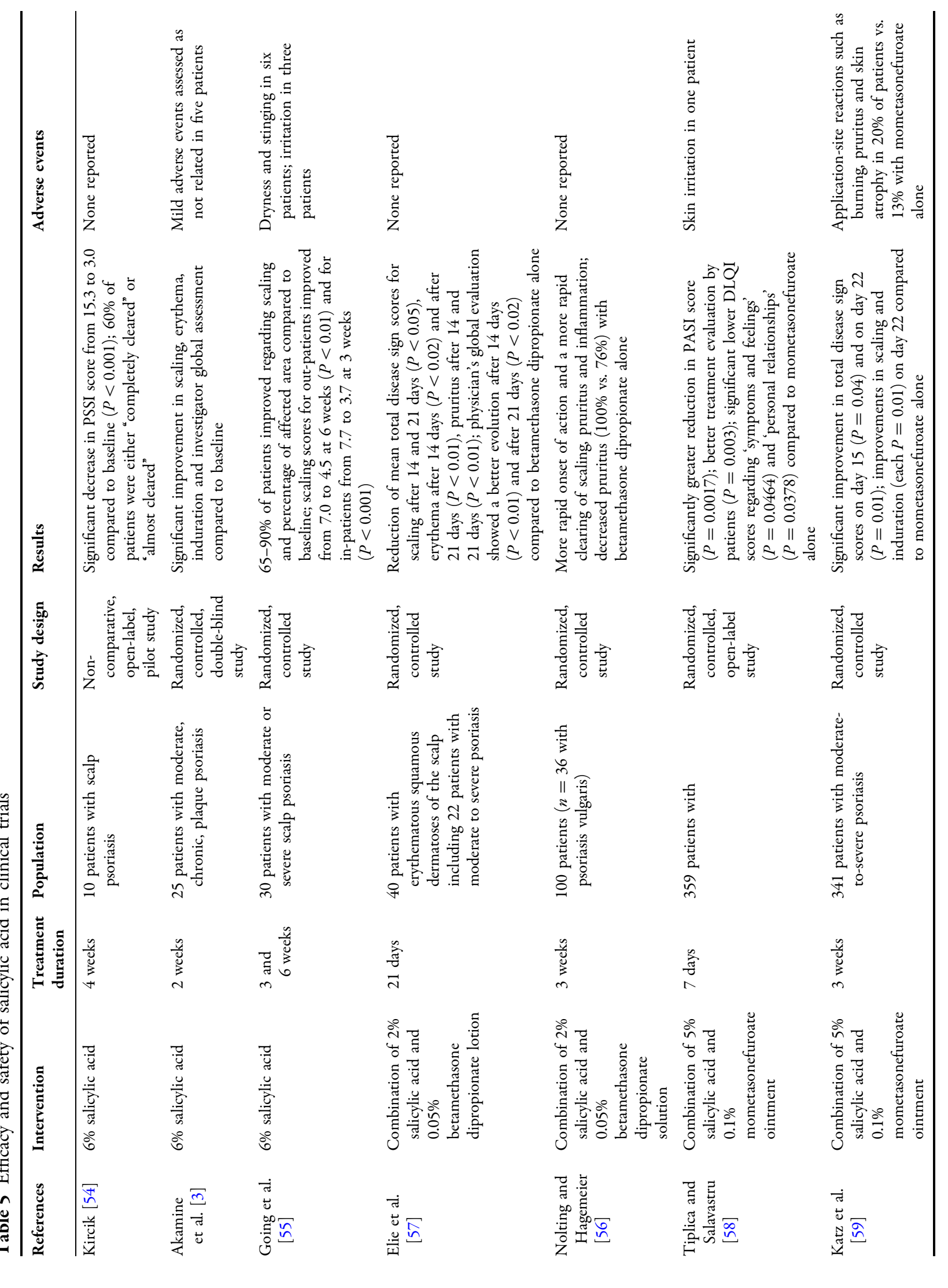




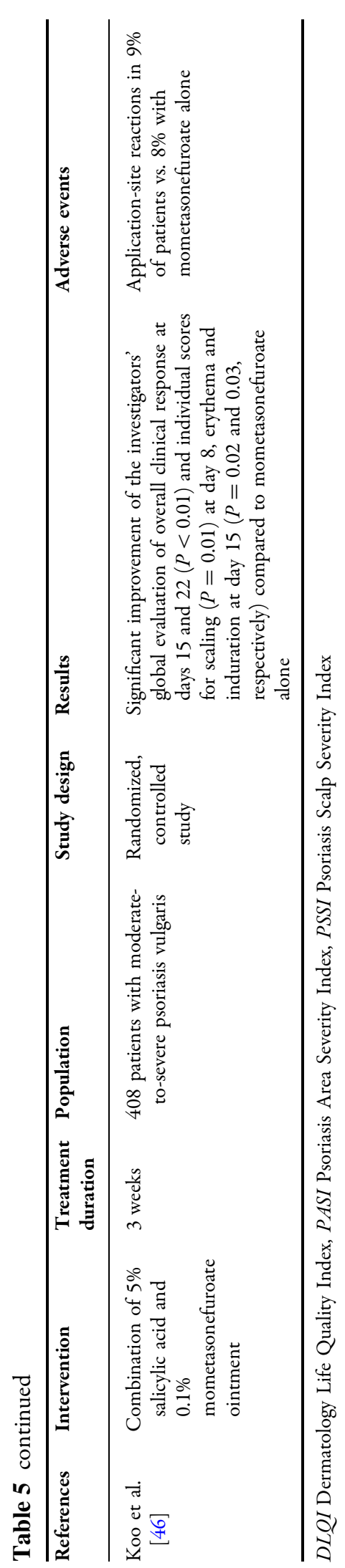

10 in-patients and 20 out-patients [55]. Inpatients were treated for 3 weeks with daily applications of $6 \%$ salicylic acid removed with a shampoo $24 \mathrm{~h}$ later. Out-patients were treated for 6 weeks with salicylic acid applied once daily and removed $12 \mathrm{~h}$ later. If progress was slow, a shampoo containing 3\% coal tar solution was substituted for the shampoo. As a result, $90 \%$ of in-patients and $65 \%$ of out-patients improved with salicylic acid regarding scaling and percentage of affected area and in one patient, the scalp cleared completely. Scaling scores for out-patients improved from baseline 7.0 to 4.5 at 6 weeks $(n=20 ; P<0.01)$ and the scores for inpatients improved from baseline 7.7 to a score of 3.7 at 3 weeks $(n=10 ; P<0.001)$. Eight of the out-patients, but none of the in-patients used the coal tar shampoo. Out-patients used less salicylic acid (27 g weekly) than in-patients (72 g weekly; see Table 5) [55].

\section{Combination Therapy}

Concerning combination therapy with the superpotent or potent corticosteroids betamethasone dipropionate and mometasonefuroate, 5 randomized controlled trials in a total of 1,248 patients were identified. Patients with psoriasis were treated for up to 3 weeks with a combination of $2 \%$ salicylic acid and $0.05 \%$ betamethasone dipropionate ( 2 studies; $n=72$ ) or $5 \%$ salicylic acid and $0.1 \%$ mometasonefuroate (3 studies; $n=519$ ). The respective control groups received the corresponding corticosteroid only. In one study, a more rapid onset of action and a more rapid clearing of scaling, pruritus, and inflammation was demonstrated with the combination [56]; whereas in another trial, the combination was statistically more effective than the respective corticosteroid alone in terms of mean total disease sign scores beginning at day 8 $(P=0.05)$ and continuing through days 15 and $22(P<0.01)[46]$. This is also reflected in the 
individual sign scores: the combination was significantly more effective than the corticosteroid alone for scaling starting on day $8(P=0.01)$, erythema $(P<0.02)$, and pruritus on day $14(P<0.01)$ as well as induration on day 15 $(P=0.03)$, with $P \leq 0.01$ for all three signs of psoriasis (erythema, induration and scaling) at day 22 [46, 57]. According to the investigators' global evaluation of overall clinical response, patients treated with the combination showed a better progress than those treated with the corticosteroid alone $(P<0.01)$ [46]. After 7 days of treatment, a significantly greater reduction of the Psoriasis Area Severity Index score was observed compared to the corticosteroid alone $(P=0.0017)$. Regarding the items of the Dermatology Life Quality Index (DLQI) questionnaire, data showed significant differences in the 'symptoms and feelings' $(P=0.0464)$ and the 'personal relationships' items $(P=0.0378)$ [58]. On the self-assessment questionnaire, patients preferred the combination over the corticosteroid alone for overall improvement of general appearance and overall improvement of psoriasis (both $P=0.03$; see Table 5) [59]. In the evaluated studies, no systemic toxicity was observed and absorption of salicylate was negligible after application of $6 \%$ to the scalp [55]. Yet application-site reactions such as dryness, irritation, burning, pruritus, and skin atrophy were reported (see Table 5) $[46,55$, $58,59]$.

\section{DISCUSSION}

This systematic literature review covering articles published from January 1983 to December 2013 identified several reviews, meta-analyses, and guidelines emphasizing that, while topical treatment with corticosteroids and vitamin D analogs currently represent first-line therapies, adjuvant therapy with topical keratolytics and emollients forms part of an integrated therapeutic concept. This is in line with the evaluated studies demonstrating specific properties of these agents that determine their role within this concept.

Regarding emollients, the evaluated studies provided some evidence that these agents play a role as a basic therapy in supporting skin care and improving the altered structure and function of the skin but without a distinct clinical response in psoriasis. Study results for urea point to a reduction of induration and/or scaling. However, the percentage of affected area was not reduced. Due to its emollient and keratolytic activity, urea may be used as a safe basic therapy leading to increased patient satisfaction. The same applies to AHAs and PHAs. However, to date, there are only a limited number of short-term studies regarding the mentioned substances, which were mostly performed in small populations without control or a clear randomization procedure and mainly using weak outcomes variables.

Salicylic acid represents the best studied keratolytic compound to date. Although salicylic acid used as monotherapy induced a significant improvement of psoriasis, as determined by the percentage of body surface area involved and/or the overall degree of erythema, induration, and desquamation [3, 55], the evidence of its efficacy as monotherapy remains controversial due to the lack of larger, high-quality trials and the absence of a placebo control which is considered a major limitation of most of the identified studies.

Only four of the studies included in this review had a placebo control, that is, a comparison with the cream vehicle. In three of these studies, treatment with the active 
substance (i.e., urea) led to a statistically significant improvement of outcome parameters compared to the vehicle [41]. In two studies, the ointment base (which was not further specified) also improved psoriasis, but to a significantly lesser extent than the pharmacologically active ingredient, urea [38, 42]. This is in line with the studies on emollients, that may represent constituents of an ointment base and that did not show any relevant reduction in the severity of psoriasis.

As regards its place in therapy, results of the current literature analysis support the use of salicylic acid as monotherapy in the treatment of scalp psoriasis and as an adjuvant agent in the treatment of other skin areas affected by psoriasis. Yet, patient adherence in scalp psoriasis is often poor due to the longer contact times required and since vehicles tend to be greasy [11]. In combination with the super-potent or potent corticosteroids betamethasone dipropionate and mometasonefuroate, evidence from several randomized controlled trials in a total of 1,248 patients demonstrates that addition of salicylic acid is beneficial in inducing a more rapid onset of action and a reduction of psoriasis determined by the percentage of body surface area involved and/or the overall degree of erythema, induration, and desquamation. The addition of salicylic acid led to a better investigators' global evaluation of overall clinical response, a more favorable evaluation by patients and significant lower DLQI scores compared to the respective corticosteroid alone.

Since treatment outcome determines treatment satisfaction, which in turn affects medication adherence, keratolytic agents such as salicylic acid may support overall adherence to the prescribed therapy. While salicylic acid at the applied concentrations was well tolerated in the evaluated studies with no signs of systemic toxicity, concerns of systemic toxicity are consistently expressed in the literature $[2,3,6$, $7,22,27]$.

\section{CONCLUSION}

Considering that descaling and/or penetration enhancement entails a distinct benefit in patients with psoriasis improving patient satisfaction and treatment adherence, there still is a need for well-designed studies on the currently available keratolytic agents but also on suitable keratolytic alternatives to salicylic acid with less potential for adverse effects. Costeffectiveness should also be addressed in future studies.

\section{ACKNOWLEDGMENTS}

Sponsorship for this study and article processing charges was funded by G. PohlBoskamp GmbH \& Co. KG, Hohenlockstedt, Germany. All named authors meet the ICMJE criteria for authorship for this manuscript, take responsibility for the integrity of the work as a whole and have given final approval for the version to be published.

Conflict of interest. Arnd Jacobi, Anke Mayer, and Matthias Augustin declare no conflict of interest.

Compliance with ethics guidelines. This review is based on previously conducted studies, and does not involve any new studies of human or animal subjects performed by any of the authors. 
Open Access. This article is distributed under the terms of the Creative Commons Attribution Noncommercial License which permits any noncommercial use, distribution, and reproduction in any medium, provided the original author(s) and the source are credited.

\section{REFERENCES}

1. Cohen SN, Baron SE, Archer CB. Guidance on the diagnosis and clinical management of psoriasis. Clin Exp Dermatol. 2012;37(Suppl 1):13-8.

2. Cordoro KM. Topical therapy for the management of childhood psoriasis: part I. Skin Therapy Lett. 2008;13:1-3.

3. Akamine KL, Gustafson CJ, Yentzer BA, et al. A double-blind, randomized clinical trial of $20 \%$ alpha/poly hydroxy acid cream to reduce scaling of lesions associated with moderate, chronic plaque psoriasis. J Drugs Dermatol. 2013;12:855-9.

4. Federman DG, Froelich CW, Kirsner RS. Topical psoriasis therapy. Am Fam Physician. 1999;59(957-62):964.

5. Sticherling M, Augustin M, Boehncke W, et al. Therapy of psoriasis in childhood and adolescence-a German expert consensus. J Dtsch Dermatol Ges. 2011;9:815-23.

6. Witman PM. Topical therapies for localized psoriasis. Mayo Clin Proc. 2001;76:943-9.

7. Menter A, Korman NJ, Elmets CA, et al. Guidelines of care for the management of psoriasis and psoriatic arthritis: section 4 . Guidelines of care for the management and treatment of psoriasis with traditional systemic agents. J Am Acad Dermatol. 2009;61:451-85.

8. Samarasekera EJ, Sawyer L, Wonderling D, Tucker R, Smith CH. Topical therapies for the treatment of plaque psoriasis: systematic review and network meta-analyses. Br J Dermatol. 2013;168:954-67.

9. Naldi L, Rzany B. Psoriasis (chronic plaque). Clin Evid (Online). 2009; pii: 1706.

10. Nast A, Boehncke WH, Mrowietz U, et al. German S3-guidelines on the treatment of psoriasis vulgaris (short version). Arch Dermatol Res. 2012;304:87-113.
11. Pauporte $\mathrm{M}$, Maibach $\mathrm{H}$, Lowe $\mathrm{N}$, et al. Fluocinoloneacetonide topical oil for scalp psoriasis. J Dermatolog Treat. 2004;15:360-4.

12. Lodén M. Role of topical emollients and moisturizers in the treatment of dry skin barrier disorders. Am J ClinDermatol. 2003;4:771-88.

13. Chiricozzi A, Chimenti S. Effective topical agents and emerging perspectives in the treatment of psoriasis. Expert Rev Dermatol. 2012;7:283-93.

14. Nast A, Boehncke W, Mrowietz U, et al. S3guidelines on the treatment of psoriasis vulgaris (English version). Update. J Dtsch Dermatol Ges. 2012;10(Suppl 2):S1-95.

15. Birgin B, Fetil E, Ilknur T, TahsinGüneş A, Ozkan S. Effects of topical petrolatum and salicylic acid upon skin photoreaction to UVA. Eur J Dermatol. 2005;15:156-8.

16. Kornhauser A, Coelho SG, Hearing VJ. Applications of hydroxy acids: classification, mechanisms and photoactivity. Clin Cosmet Investig Dermatol. 2010;3:135-42.

17. Augustin M, Holland B, Dartsch D, Langenbruch A, Radtke MA. Adherence in the treatment of psoriasis: a systematic review. Dermatology. 2011;222:363-74.

18. Kinney MA, Feldman SR. What's new in the management of psoriasis? G Ital Dermatol Venereol. 2009;144:103-17.

19. Carroll CL, Clarke J, Camacho F, Balkrishnan R, Feldman SR. Topical tacrolimus ointment combined with $6 \%$ salicylic acid gel for plaque psoriasis treatment. Arch Dermatol. 2005;141:43-6.

20. Ryan S. Psoriasis: characteristics, psychosocial effects and treatment options. $\mathrm{Br} \mathrm{J}$ Nurs. 2008;17:284-90.

21. Fluhr JW, Cavallotti C, Berardesca E. Emollients, moisturizers and keratolytic agents in psoriasis. Clin Dermatol. 2008;26:380-6.

22. Lebwohl M. The role of salicylic acid in the treatment of psoriasis. Int $\mathrm{J}$ Dermatol. 1999;38:16-24.

23. Finlay A. Emollients as adjuvant therapy for psoriasis. J Dermatol Treat. 1997:25-27.

24. Hendriks AGM, Keijsers RRMC, de Jong EMGJ, Seyger MMB, van de Kerkhof PCM. Combinations of classical time-honoured topicals in plaque psoriasis: a systematic review. J Eur Acad Dermatol Venereol. 2013;27:399-410. 
25. Childhood psoriasis: often favorable outcome. Prescrire Int. 2009;18:275.

26. Dawn A, Yosipovitch G. Treating itch in psoriasis. Dermatol Nurs. 2006;18:227-33.

27. Raut AS, Prabhu RH, Patravale VB. Psoriasis clinical implications and treatment: a review. Crit Rev Ther Drug Carrier Syst. 2013;30:183-216.

28. Tidman MJ. Improving outcomes in patients with psoriasis. Practitioner. 2013;257(27-30):3.

29. Berne B, Blom I, Spångberg S. Enhanced response of psoriasis to UVB therapy after pretreatment with a lubricating base. A single-blind controlled study. Acta Derm Venereol. 1990;70:474-7.

30. Boyvat A, Erdi H, Birol A, Gurgey E. Interaction of commonly used emollients with photochemotherapy. Photodermatol Photoimmunol Photomed. 2000;16:156-60.

31. Kristensen B, Kristensen O. Topical salicylic acid interferes with UVB therapy for psoriasis. Acta Derm Venereol. 1991;71:37-40.

32. Lebwohl M, Martinez J, Weber P, DeLuca R. Effects of topical preparations on the erythemogenicity of UVB: implications for psoriasis phototherapy. J Am Acad Dermatol. 1995;32:469-71.

33. van Duijnhoven, Mirella WFM, Hagenberg R, et al. Novel quantitative immunofluorescent technique reveals improvements in epidermal cell populations after mild treatment of psoriasis. Acta Derm Venereol. 2005;85:311-7.

34. Rim JH, Jo SJ, Park JY, Park BD, Youn JI. Electrical measurement of moisturizing effect on skin hydration and barrier function in psoriasis patients. Clin Exp Dermatol. 2005;30:409-13.

35. Tanghetti EA. An observation study evaluating the treatment of plaque psoriasis with tazarotene gels, alone and with an emollient and/or corticosteroid. Cutis. 2000;66:4-11.

36. Watsky KL, Freije L, Leneveu MC, Wenck HA, Leffell DJ. Water-in-oil emollients as steroidsparing adjunctive therapy in the treatment of psoriasis. Cutis. 1992;50:383-6.

37. Singh S, Gopal J, Mishra RN, Pandey SS. Topical $0.05 \%$ betamethasone dipropionate: efficacy in psoriasis with once a day vs. twice a day application. Br J Dermatol. 1995;133:497-8.

38. Hagemann I, Proksch E. Topical treatment by urea reduces epidermal hyperproliferation and induces differentiation in psoriasis. Acta Derm Venereol. 1996;76:353-6.
39. Pan M, Heinecke G, Bernardo S, Tsui C, Levitt J. Urea: a comprehensive review of the clinical literature. Dermatol Online J. 2013;19:20392.

40. Gip L, Lundberg M. A double-blind controlled trial of a new antipsoriatic cream containing urea and sodium chloride. CurrTher Res. 1985;37:797-804.

41. Fredriksson T, Lundberg M. A blind controlled comparison between a new cream (" $12+12$ "), its vehicle and salicylic acid in petrolatum on psoriatic plaques. Curr Ther Res. 1985;37:805-9.

42. Lakshmi PK, Bhaskaran S. Phase II study of topical niosomal urea gel-an adjuvant in the treatment of psoriasis. Int J Pharm Sci Rev Res. 2011;7(1):1-7.

43. Vena GA, Cassano N, Agnusdei CP, et al. Treatment of psoriasis vulgaris with calcipotriol betamethasone dipropionate combination followed by calcipotriol and assessment of the adjuvant basic use of ureabased emollients. Eur J Inflamm. 2005;3:37-41.

44. Shemer A, Nathansohn N, Kaplan B, Weiss G, Newman N, Trau H. Treatment of scalp seborrheic dermatitis and psoriasis with an ointment of $40 \%$ urea and $1 \%$ bifonazole. Int $\mathrm{J}$ Dermatol. 2000;39:532-4.

45. Taube KM, Fiedler H, Wohlrab W, Wozniak KD. Untersuchungen zur Kurzzeitbehandlung der Psoriasis vulgaris mit Dithranol unter Harnstoffzusatz. Dermatol Monatsschr. 1985;171:650-3.

46. Koo J, Cuffie CA, Tanner DJ, et al. Mometasonefuroate $\quad 0.1 \%$-salicylic acid $5 \%$ ointment one-17,21-dipropionate in the treatment of erythematous squamous dermatoses. J Int Med Res. 1983;11:108-12.

47. Berardesca E, PieroVignoli G, Distante E, Rona C. Effects of glycolic acid on psoriasis. Clin Exp Dermatol. 1998;23:190-1.

48. Kostarelos K, Teknetzis A, Lefaki I, Ioannides D, Minas A. Double-blind clinical study reveals synergistic action between alpha-hydroxy acid and betamethasone lotions towards topical treatment of scalp psoriasis. J Eur Acad Dermatol Venereol. 2000;14:5-9.

49. Chan CS, Van Voorhees, Abby S, et al. Treatment of severe scalp psoriasis: from the Medical Board of the National Psoriasis Foundation. J Am Acad Dermatol. 2009;60:962-71.

50. Cox NH, Sharpe G. Emollients, salicylic acid and ultraviolet erythema. Lancet. 1990;335:53-4.

51. Fetil E, Ozka S, Soyal MC, Ilknur T, Erdem Y, Güneş AT. Effects of topical petrolatum and salicylic acid 
on the erythemogenicity of UVB. Eur J Dermatol. 2002;12:154-6.

52. Peters BP, Weissman FG, Gill MA. Pathophysiology and treatment of psoriasis. Am J Health Syst Pharm. 2000;57:645-59.

53. Ortonne J, Chimenti S, Luger T, Puig L, Reid F, Trüeb RM. Scalp psoriasis: European consensus on grading and treatment algorithm. J Eur Acad Dermatol Venereol. 2009;23:1435-44.

54. Kircik L. Salicylic Acid 6\% in an ammonium lactate emollient foam vehicle in the treatment of mild-tomoderate scalp psoriasis. J Drugs Dermatol. 2011;10:270-3.

55. Going SM, Guyer BM, Jarvie DR, Hunter JA. Salicylic acid gel for scalp psoriasis. Clin Exp Dermatol. 1986;11:260-2.
56. Nolting S, Hagemeier HH. Therapie erythrosquamöser Dermatosen. BetamethasonDipropionat plus Salizylsäure im Vergleich $\mathrm{zu}$ Betamethason-Dipropionat-Lösung. Fortschr Med. 1983;101:1679-83.

57. Elie R, Durocher LP, Kavalec EC. Effect of salicylic acid on the activity of betamethas. Curr Ther Res. 1983;34:459-68.

58. Tiplica G, Salavastru CM. Mometasonefuroate $0.1 \%$ and salicylic acid 5\% vs. mometasonefuroate $0.1 \%$ as sequential local therapy in psoriasis vulgaris. J Eur Acad Dermatol Venereol. 2009;23:905-12.

59. Katz H, Tanner D, Cuffie CA, et al. A comparison of the efficacy and safety of the combination mometasonefuroate $0.1 \% /$ salicylic acid $5 \%$ ointment with each of its components in psoriasis. J Dermatolog Treat. 1998;9:151-6. 